\title{
Conte et morale(s), du XVII à aujourd'hui, sous la direction de Jean MAINIL et Jean-Paul SERMAIN
}

\section{Roberta Sapino}

\section{(2) OpenEdition \\ Journals}

\author{
Edizione digitale \\ URL: https://journals.openedition.org/studifrancesi/12288 \\ DOI: 10.4000/studifrancesi. 12288 \\ ISSN: 2427-5856

\section{Editore} \\ Rosenberg \& Sellier

\section{Edizione cartacea} \\ Data di pubblicazione: 1 avril 2018 \\ Paginazione: 181-182 \\ ISSN: 0039-2944 \\ Notizia bibliografica digitale \\ Roberta Sapino, «Conte et morale(s), du xvIl à aujourd'hui, sous la direction de Jean mainIL et Jean-Paul \\ SERMAIN», Studi Francesi [Online], 184 (LXII | I) | 2018, online dal 04 juillet 2018, consultato il 18 \\ novembre 2021. URL: http://journals.openedition.org/studifrancesi/12288 ; DOI: https://doi.org/ \\ 10.4000/studifrancesi.12288
}

Questo documento è stato generato automaticamente il 18 novembre 2021.

\section{(c) ()) (9)}

Studi Francesi è distribuita con Licenza Creative Commons Attribuzione - Non commerciale - Non opere derivate 4.0 Internazionale. 


\title{
Conte et morale(s), du XVII ${ }^{e} \grave{a}$ aujourd'hui, sous la direction de Jean MAINIL et Jean-Paul SERMAIN
}

\author{
Roberta Sapino
}

\section{NOTIZIA}

Conte et morale(s), du XVII à aujourd'hui, sous la direction de Jean MAINIL et Jean-Paul SERMAIN, Ellug, 2016, «Féeries» 13, 296 pp.

1 Arrivata al tredicesimo anno di pubblicazione, la rivista «Féeries» declina la sua tradizione di studi sul conte merveilleux in un numero dedicato alle implicazioni morali della (e nella) scrittura, curato da Jean MAINIL et Jean-Paul SERMAIN. Un avant-propos di poche righe (Jean Paul Sermain et Jean Mainil, p. 9) apre la via ai tredici contributi che compongono la sezione tematica della rivista, la cui coerenza è messa in luce in maniera particolarmente efficace nel primo articolo, firmato da Mainil (Conte et morale, ou Les nouveaux habits de la Moralité, pp. 11-25), che funge anche da vera e propria introduzione. La consueta presentazione dei contributi raccolti nel volume è preceduta da una breve analisi non solo degli intenti morali espressi da Charles Perrault, ma anche delle sorti che i contes incontrarono nei secoli seguenti. Tra riscritture, riappropriazioni e rimaneggiamenti, il conte rivela fin dalle origini una malleabilità sorprendente, che l'insieme del volume ben valorizza.

2 Proprio la versatilità intrinseca al conte permette a Hélène MERLIN-KAJMAN non solo di sviluppare una riflessione acuta sul valore allegorico tradizionalmente attribuito ai personaggi animali e sui suoi possibili (e talvolta poco riusciti) rovesciamenti, ma anche di scendere "nella gola del lupo" per riemergerne autrice di una serie di fiabe basate sulla riscrittura di alcuni episodi di La Fontaine (Écrire dans la gueule du loup, pp. 27-45). L'introduzione di una cornice narrativa nuova, in cui il lettore può osservare le reazioni di un bambino rispetto al racconto, si delinea come la realizzazione di un intento 
morale e politico preciso: lottare contro il sentimento di impotenza e il cinismo dilaganti e favorire la riapertura a un vero e proprio dialogo affettivo.

3 In Poétique du récit: vie morale et sens moral dans les "Contes" de Perrault (pp. 47-64), JeanPaul SERMAIN si interroga sulla «legge chimica» che nel conte de fée garantisce il funzionamento di un sistema testuale basato sulla libertà di interpretazione di elementi spesso contraddittori. A partire dai testi di Perrault, analizzati con particolare attenzione ai seuils (le introduzioni), Sermain introduce una riflessione più ampia sui rapporti complessi tra senso razionale e senso morale in letteratura.

4 Il saggio di Ute HEIDMANN, intitolato Reconfigurer les contes pour moraliser autrement. Fuseau, quenouille de verre et pantoufle de verre (pp. 65-85), amplia la prospettiva dall'autore singolo al campo letterario. A partire dallo studio dei rapporti testuali che legarono Perrault non solo ad Apuleio (di cui reinterpretò la fabella di Psiche), ma anche a Marie-Jeanne Lhéritier (autrice di un volume di Cuvres meslées) e a Basile (la cui Gatta Cenerentola offrì parte della materia per Cendrillon ou la petite pantoufle de verre), Ute Heidmann mette in luce il gioco dialogico che fonda e sostiene lo sviluppo del conte francese.

5 Con Pierre-Emmanuel Moog (L'interdit salutaire à travers deux cas merveilleux: "Cendrillon" (Perrault) et "Les six cygnes" (Grimm), pp. 87-115) ci si allontana in parte dal territorio francese per compiere una breve incursione nel mondo dei fratelli Grimm. L'analisi comparativa di due opere scritte in ambiti geograficamente, culturalmente e cronologicamente piuttosto lontani induce Moog a elaborare osservazioni di carattere generale riguardo alla costruzione del conte: in particolare, il divieto, soprattutto quando enunciato in maniera trasgressiva rispetto allo schema di Propp, avrebbe funzione salvifica nei riguardi del personaggio al quale è diretto. Al di là delle conclusioni proposte, l'articolo apre una serie di piste di ricerca promettenti in vista di progetti di più ampio respiro.

6 In Les persifleurs moralistes: Moncrig, Duclos et Crébillon témoins de leur temps, Emmanuelle SEMPÈRE (pp. 117-130) ci porta nella Francia della metà del XVIII secolo, e in particolare, in quegli ambienti mondani in cui il discorso si fa affabulazione - persiflage appunto. Ripercorrendo le tappe che portarono da un lato alla diffusione del termine, dall'altro all'appropriazione del fenomeno da parte della letteratura, Emmanuelle Sempère osserva come l'evocazione critica del linguaggio dei persifleurs costituisca per gli autori studiati non tanto un tema quanto un mezzo per riflettere sulla funzione della parola all'interno della società.

7 Stéphanie BERNIER-TOMAS si concentra su Désinvolture morale et revendications féministes dans le conte en vers des Lumières (pp. 131-144). La rivalutazione dei temi licenziosi precedentemente evitati per rispetto della bienséance introduce nella letteratura del XVIII secolo un orizzonte provocatorio dal quale il conte non è immune, e che in testi come Ce qui plait aux dames di Voltaire o Le Prince des feuilles di Mme de Murat trova alcune delle sue declinazioni più raffinate. Liberato dalle convenzioni dell'estetica galante, il conte in versi del Settecento rivaluta lo spirito epicureo e il principio di piacere in quanto orizzonti fondamentali nella ricerca del bonheur tanto per gli uomini quanto per le donne, favorendo così la riflessione sulla posizione sociale della figura femminile.

8 La guérison par l'exemple: morale, politique et exemplarité dans les "Mille et Une Nuits" et leur hypertexte è il titolo del contributo di Dominique JulLIEN (pp. 145-163), in cui l'analisi 
delle notti 145-150 nella traduzione di Burton è seguita dallo studio di un testo che nelle Mille e una Notte trova un modello intertestuale importante: Les Mystères de Paris di Eugène Sue, e in particolare l'episodio di Pique-Vinaigre. Nei due testi il legame tra la dimensione morale, pedagogica e terapeutica è particolarmente stretto e non privo di implicazioni di natura politica.

In Les anges déchus qui font rire (pp. 165-182), Aboubakr CHARÄ̈BI risale alle fonti arabe, turche e persiane dell'Histoire des deux frères génies Adis et Dahy di Pétits de La Croix, testo in cui l'ironia affianca un'interrogazione morale riconducibile tanto al cristianesimo quanto all'islam, in virtù del sostrato orientale comune alle due religioni. Se nell'articolo Charaïbi sceglie di soffermarsi su tre categorie di narrazioni tematicamente o strutturalmente affini al testo di Pétits de La Croix, la conclusione lascia intravedere la possibilità di espandere la ricerca seguendo assi diversi e ugualmente fecondi.

Nel XVIII secolo illuminista, ricorda Magali fourgnaud in Critique et morale dans "Le Taureau blanc" de Voltaire (1774) (pp. 183-197), il termine "morale" è associato all'esercizio autonomo del giudizio secondo ragione, e per questo arriva pressoché a coincidere con il concetto di "filosofia". In questo contesto, Le Taureau blanc, l'ultimo conte oriental scritto da Voltaire, emerge come una critica accesa - e a tratti paradossale - di alcuni episodi biblici, i cui aspetti soprannaturali sono ricondotti alla dimensione letteraria, quando non alla più terrena superstizione.

Il contributo di Françoise GERVREY (La «morale expérimentale» du conte: l'exemple des “Contes sages et fous" d'Angélique Desjardins (1787), pp. 199-216) ripercorre analiticamente la genesi e lo sviluppo della raccolta di contes di Angélique Desjardins, mostrando i rapporti complessi che essa instaura con il contesto letterario e filosofico del suo tempo. Il gusto per il meraviglioso e l'intento moralizzatore emergono come i due poli entro i quali prende forma una scrittura che arriva a competere con il romanzo d'educazione.

12 Le dichiarazioni degli autori riguardo alle loro opere sono talvolta ingannevoli, e il contributo di Joëlle LÉGERET ne propone una dimostrazione pregnante. Ponendosi in aperta contraddizione con ciò che i fratelli Grimm scrissero in apertura al secondo volume delle Kinder- und Hausmärchen, la studiosa mette in luce come il dialogismo intertestuale sia una componente fondamentale dell'impresa letteraria dei fratelli Grimm. L'analisi di Sneewittchen consente di identificare in Albert Ludwig Grimm uno degli interlocutori tanto importanti quanto dissimulati in rapporto ai quali i due fratelli concepirono la loro scrittura (Contes pour enfants ou livre d'éducation? Albert Ludwig Grimm et les "frères Grimm» autour de "S(ch)neewittchen", pp. 217-234).

13 L'ultimo articolo affronta una traduzione-riscrittura dei contes di Charles Perrault che ebbe particolare fortuna: quella realizzata nel 1897 da Ekaterina Ursinovitch. Pur non costituendo la prima trasposizione in russo dei testi di Perrault, l'operazione di Ursinovitch è interessante in virtù degli importanti rimaneggiamenti che essa presenta, volti da un lato ad avvicinare ogni narrazione alla cultura del nuovo pubblico cui è destinata, dall'altro a consolidare la portata didattica della raccolta nel suo insieme (Véronika ALTACHINA, Une réécriture russe des contes de Charles Perrault, pp. 235-249).

14 Chiudono il volume un intenso Hommage à René Démoris (1935-2016) firmato da Anne DEFRANCE (pp. 251-255), una serie di Comptes rendus critiques (pp. 257-268), gli abstract in 
francese e inglese degli articoli (pp. 269-280) e un'utile lista dei sommari di tutti i numeri della rivista pubblicati finora (pp. 281-296). 